\title{
The Puerto Rico Photonics Institute
}

Jonathan Friedman

Jonathan S. Friedman, "The Puerto Rico Photonics Institute," Proc. SPIE 9289, 12th Education and Training in Optics and Photonics Conference, 92890A (17 July 2014); doi: 10.1117/12.2070283

SPIE Event: 12th Education and Training in Optics and Photonics Conference, SPIE. 2013, Porto, Portugal 


\title{
The Puerto Rico Photonics Institute
}

Jonathan S. Friedman, $\mathrm{PhD}$

Puerto Rico Photonics Institute

School of Environmental Affairs

Universidad Metropolitana

San Juan PR 00926-1150

USA

\begin{abstract}
We have founded the Puerto Rico Photonics Institute (PRPI) in the Barceloneta, Puerto Rico campus of the Universidad Metropolitana. PRPI is established to provide opportunities in education, training and research and is unique in Puerto Rico. There are two initial focus areas of research and education: aerospace photonics and remote sensing. In particular, we will conduct studies and research and development in two particular fields: laser gyroscopes and similar technologies, and atmospheric remote sensing. PRPI has established local collaborations with the Arecibo Observatory and Honeywell Aerospace. Outside of Puerto Rico, PRPI collaborators include the University of Central Florida (CREOL), University of Arizona (OSC), University of Dayton (UD), Georgia Institute of Technology (GT), Scientific Solutions, Inc. (SSI), Atmospheric and Space Technology Research Associates (ASTRA), and the MIT Draper Laboratory. These organizations will help PRPI to: 1) establish its curriculum, provide research opportunities for PRPI students, 2) participate in faculty exchange programs, and 3) build its own research and development programs. PRPI will have educational and training programs for both Associate and Masters degrees, as well as a Certificate in Optics and Photonics for undergraduate science and engineering majors and professional engineers. PRPI is supported by UMET's parent institution, the Ana G. Mendez University System (SUAGM), the Puerto Rico Science, Technology and Research Trust (PRST), and the Puerto Rico Industrial Development Company (PRIDCO).
\end{abstract}

\section{Introduction}

The Puerto Rico Photonics Institute (PRPI) is conceived as an education, training, and research institute in optics and photonics. It will inspire students to careers in STEM fields, and it will provide hands-on and theoretical instruction and research opportunities for students and professionals to prepare them for industry or scholarship in optics and photonics research and development. PRPI is an institute of the Universidad Metropolitana (UMET), which is part of the Ana G. Mendez University System (SUAGM), and is located in Barceloneta, Puerto Rico, USA.

12th Education and Training in Optics and Photonics Conference, edited by

Manuel F. P. C. Martins Costa, Mourad Zghal, Proc. of SPIE Vol. 9289, 92890A

(C) 2014 SPIE, OSA, IEEE, ICO · doi: 10.1117/12.2070283

Proc. of SPIE Vol. 9289 92890A-1 
PRPI addresses part of the need for growth in science, technology, engineering and mathematics (STEM) education, research and development, and the fact that Hispanics and women are profoundly under-represented in these fields. In particular, it aims to fill a large gap in science and engineering educational and career opportunities in Puerto Rico. Puerto Rico offers very limited programs that teach optics (optometry), and none for photonics. Industries that depend on optics and photonics either carry out their research and development work elsewhere, recruit scientists and engineers from outside of Puerto Rico, and/or train their Puerto Rico employees in house. There is currently no home grown photonics industry in Puerto Rico, in spite of the economic potential and myriad technology possibilities it offers.

The 1998 National Academy of Sciences report entitled "Harnessing Light: Optical Science and Engineering for the 21st Century," states, "... optics courses in most universities are taught in several science departments, as well as engineering departments and schools of medicine. As a result of such organizational structures as these-in spite of, or perhaps because of, its pervasive importance - optics tends to be an orphan, owned by no one" [Shank et al., 1998]. This is changing in many places in the USA, as our partner universities can attest. In Puerto Rico, the situation is even more dire, yet many state-of-the-art and up-and-coming technologies in communications, medicine, computation, space technologies, remote sensing, tracking, and manufacturing require sophisticated and formalized knowledge of optics and photonics.

PRPI's core program is to educate pre- and post-baccalaureate students, engineers and other high technology industry professionals in optical science, engineering and technology. It will consist of laboratory and classroom facilities at the Barceloneta Campus of the Universidad Metropolitana (UMET) in north-central Puerto Rico. PRPI will educate, train, and provide real-world experience to prepare students to contribute throughout the broad spectrum of the optical sciences, but with a particular focus in two areas of particular interest in Puerto Rico: remote sensing and aerospace photonics. We will use our close association with the Arecibo Observatory to provide training, testing and development in coordination with their atmospheric optics program, and in aerospace we will develop with Honeywell Corporation in Aguadilla, PR a Masters' degree program in aerospace photonics. The Barceloneta campus is co-located with a new Science Park, which currently consists of a 3700 square meter business incubator, and there are plans to develop a graduate school in astronomy and space physics on the site.

\section{Institute Structure}

Figure 1 is a flowchart of how we envision the PRPI institutional structure. Elements of PRPI include the curriculum, which is aimed at both technical training and advanced undergraduate and graduate level education. The chart mentions "Partner" and "Collaborator" institutes. These do not imply formal agreements (although some exist), but rather the nature of associations between PRPI and the various institutes mentioned. Collaborator institutes will help extensively in establishing the curriculum through implementation and adaptation of existing courses to PRPI's needs. The University of Arizona College of Optical Sciences (OSC) will contribute to this development at all 
levels, while other partners will be involved at advanced levels. As one example of an advanced course, PRPI will adapt the undergraduate lidar program successfully implemented by the Georgia Institute of Technology at Agnes Scott College in Atlanta. The University of Central Florida will provide a conduit for PRPI students and graduates to move into $\mathrm{PhD}$ and other research programs. They have offered extensive collaboration at all levels of institutional development. Partner institutes are more on the side of R\&D, particularly instrument development and commercial applications of research done at or with PRPI. These include MIT Draper Laboratories and the National Science Foundation, who are supporting current research projects, and the Puerto Rico Science Trust (PRST) and the Puerto Rico Industrial Development Company (PRIDCO), who are providing foundational funds along with SUAGM. (The division between "Partners" and "Collaborators" is somewhat artificial, as there is much crossover.)

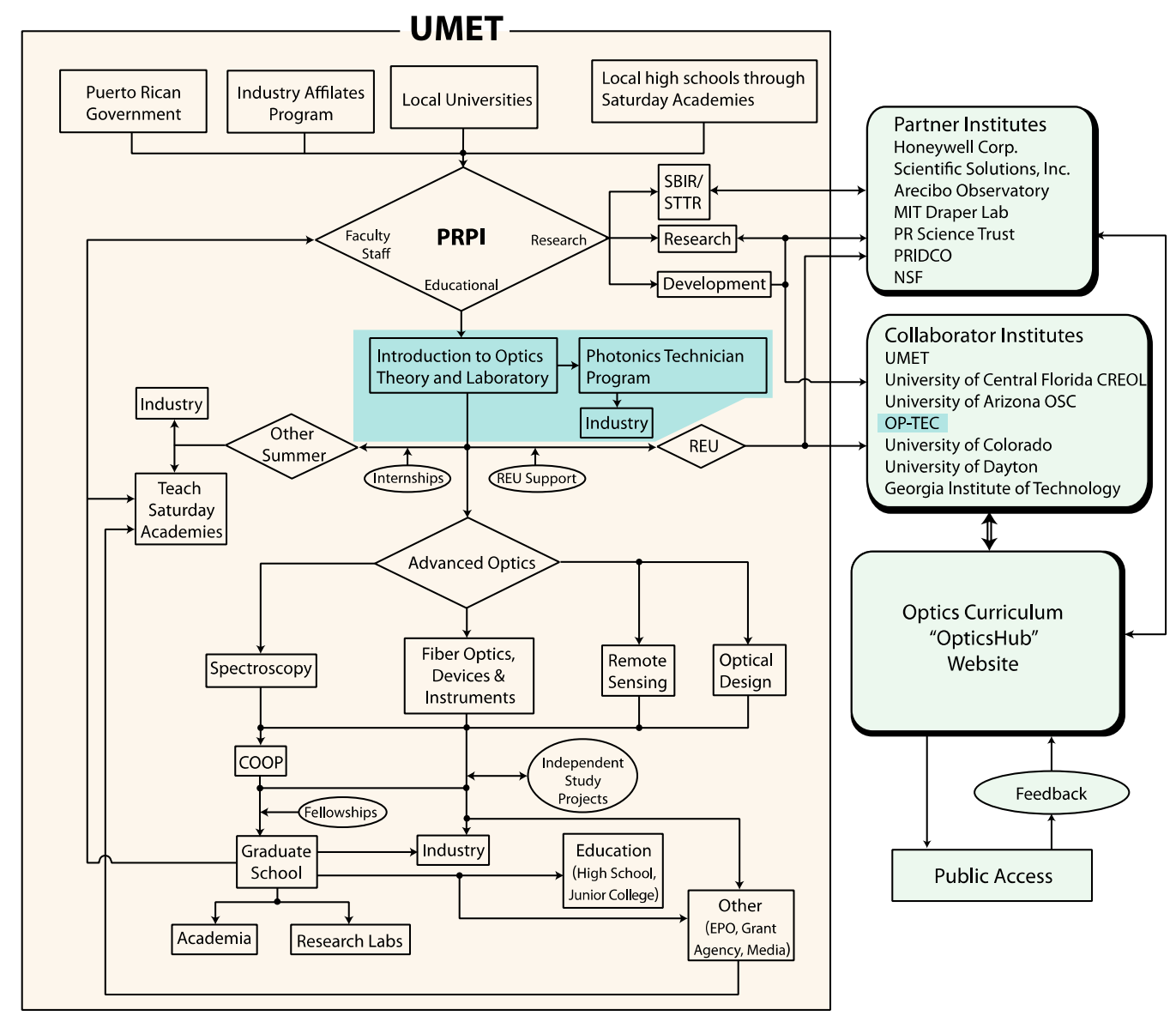

Figure 1. Organizational flowchart of PRPI. The light blue blocks highlight PRPI's training program for Associate Degrees and Certificates.

Research is fundamental to PRPI's program. Students will be required to undertake research projects, and for this purpose, PRPI will provide funding support for these students to participate in summer research experience (REU) at our collaborator and partner institutes. We will also encourage (and help) students to apply to REU programs 
at other institutes. PRPI will carry out its own research programs. First, through a Major Research Instrumentation Grant from the US National Science Foundation, PRPI is substantially upgrading the Doppler resonance lidar at the Arecibo Observatory. As a result, PRPI has inherited the equipment being replaced at Arecibo. These instruments will be used to help PRPI establish its own lidar remote sensing program, and PRPI will undertake a research and development program to advance lidar technology for Arecibo and ultimately elsewhere. PRPI faculty already collaborate closely with the NSF-funded CEDAR Lidar Consortium Technology Center (CRRL/CTC) based at the University of Colorado-Boulder. This relationship will foment technology development for lidar. It also represents graduate school opportunities at the University of Colorado and elsewhere for students who augment their undergraduate studies by taking courses at PRPI.

Other PRPI activities include participation in the development of an optics/photonics curriculum hub at OSC, creation of 14-week Saturday Academy programs for high school students in Puerto Rico, and technology development support for building a photonics industry base in Puerto Rico and an Industry Affiliates program for PRPI.

\section{Curriculum and Educational Philosophy}

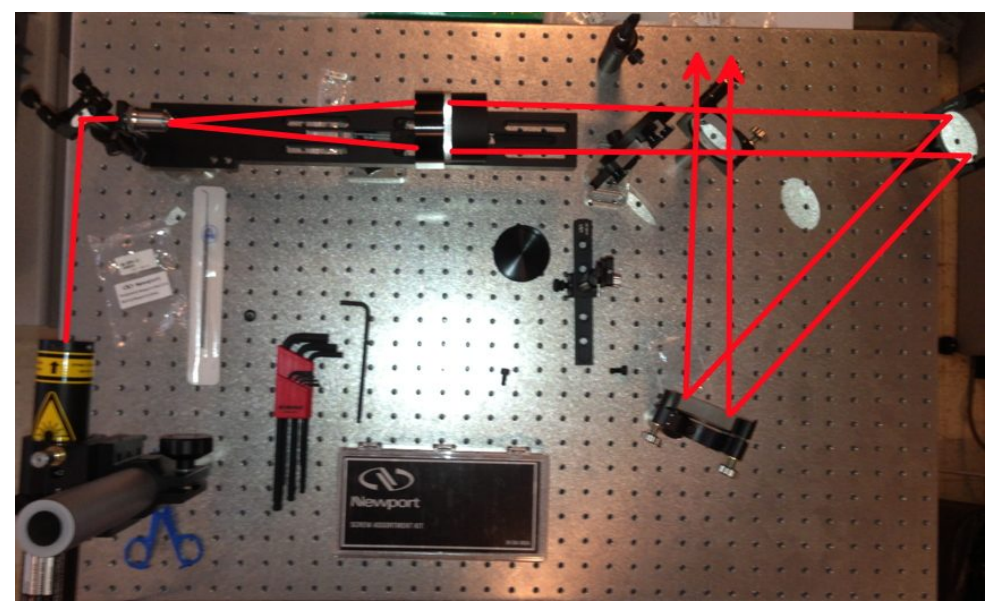

Figure 2. Layout and beam path of a ring interferometer, the basis of a laser gyroscope.

PRPI's educational philosophy is based on the fact that optics is a hands-on field of research. Students must be both competent in theory and laboratory skills in order to contribute to advancing optics and photonics science and technology. For this reason, all of our courses will be laboratory based. Our Certificate program, leading to an Associate Degree program, is based on materials from OP-TEC (www.op-tec.org), an NSF-funded National Center for photonics education and training. OP-TEC's program, starting with "Fundamentals of Light and Lasers". Students will move from the basics of handling optics and optical principles to more advanced techniques of fiber splicing, instrument and device operation, calibrations, imaging, physical optics, and more.

PRPI's higher level offerings will also revolve around the laboratory. In the Fiber Optic Devices and Instruments course, we will build the laboratory around the laser gyroscope. On the way to learning about the gyro, students will learn the basics of optical fibers and 
guided-wave transmission. The topics will include spatial modes, dispersion, gain in fibers, and information transmission. They will also be introduced to interferometers, as this is also fundamental to understanding the operation of the gyro.

The PRPI Masters-level curriculum reflects its emphasis on remote sensing and aerospace photonics. After completing an introductory course, students will have choices between Spectroscopy, Fiber Optic Devices and Instruments, Remote Sensing, and Optical Design. These broader topics will concentrate their efforts to topics that apply to the focus areas. For example, spectroscopy is fundamental to remote sensing and biophotonics, as phenomena such as fluorescence, Rayleigh and/or Raman scattering, and absorption are all employed in gleaning information from remote or microscopic targets. The Optical Design course is the least specific, but this is an area of broad and growing application as optical systems become more complex.

Having completed these courses, students will have strong qualifications to participate in research and instrument development activities. Undergraduates from Puerto Rican universities who earn a concentration in optics and photonics at PRPI will be well qualified for careers in research or industry, or continuing on to graduate school. Those who continue in the Masters' degree program will be well on their way to becoming independent researchers. As the research aspects of their degrees will be completed in Puerto Rico, we hope that this program will become a source of high technology entrepreneurs, and in so doing contribute to Puerto Rico's economic future. Some students will continue on to PhDs, some at PRPI partner institutes. Those young researchers will be the foundation of future scientific research at Puerto Rican universities.

\section{Funding Sources and Initial Directions}

Initial funding for PRPI is coming from a number of sources. SUAGM is investing $\$ 1.5 \mathrm{M}$ to build the PRPI institute, a building with 5500 square feet $\left(\sim 500 \mathrm{~m}^{2}\right)$ of space adjacent to the Barceloneta Science Park high-technology industry incubator site on the local SUAGM campus. The site includes infrastructure such as utilities and communications, and it includes security and maintenance services. UMET supports the institute director and essential personnel, such as administrative and technical staff. PRPI has received foundational grants of $\$ 273,000$ and $\$ 210,000$ from PRIDCO and PRST. These provide seed funding for research students, faculty and technical support, as well as laboratory and educational infrastructure.

PRPI collaborators and partners will also contribute by supporting PRPI students in research programs, through donations and loans of laboratory equipment, and with faculty time to interact with PRPI faculty for curriculum and research development. Research initiatives include collaborating with the CRRL/CTC on an NSF-supported project to improve the sensitivity of the Doppler resonance lidar at Arecibo, and we have plans for a collaboration with GT on an undergraduate lidar program similar to the one at Agnes Scott College. With support from MIT Draper Laboratory, PRPI is already developing the interferometry/laser gyro laboratory as part of an effort to develop new applications for laser gyros. PRPI has a collaborative with SSI and ASTRA to develop low-cost, ground-based Space Weather observation stations. Finally, we look forward to 
our initiative with Honeywell Aerospace for a Masters' program in aerospace photonics to bear fruit, which will both expand the faculty at PRPI and opportunities for its students.

\section{Summary}

We have established the Puerto Rico Photonics Institute at the Universidad Metropolitana Barceloneta Campus in Puerto Rico. This program is unique in Puerto Rico, as optics and photonics are not part of any local university curriculum. PRPI will teach and train students at levels ranging from high school through post-graduate, and engage them in research and development opportunities. We intend to expand the foundation of opportunities in high technology within Puerto Rico and to seed new industries born from within, rather than brought in from outside.

PRPI has developed relationships with top programs in optics and photonics at US universities and institutes including the University of Central Florida, University of Arizona, OP-TEC, University of Dayton, the Georgia Institute of Technology, and MIT Draper Laboratories. These will help PRPI to quickly build and implement an advanced curriculum, and they will provide research and post-graduate opportunities for PRPI students and graduates. They will also provide expertise and in-kind support. PRPI has three industry partners, Scientific Solutions, Inc., ASTRA, and Honeywell Aerospace. They will participate in the two research focus areas at PRPI, aerospace photonics and remote sensing. All of these collaborations and partnerships will lead to funding and research opportunities that will help PRPI grow.

PRPI's foundational funding comes from the Ana G. Mendez University System, as well as Puerto Rico government agencies. We are working strenuously to expand this portfolio and anticipate success in the coming months. We anticipate initial courses and activities to begin in October 2013. 